\title{
A Multiple-Sink Model for Decreasing the Energy Hole Problem in Large-Scale Wireless Sensor Networks
}

\author{
Mehrdad Ahadi and Amir Masoud Bidgoli
}

\begin{abstract}
The received data by the nodes of wireless sensor networks (WSNs) should be sent to the sink (base station) for performing calculations and making the right decisions. Therefore, the density of data packets increases near the sink and as a result, the energy of nearby nodes is depleted more rapidly. This phenomenon is called "Energy-Hole". Destruction of nodes in the proximity of sink is followed by disconnection of other nodes' links with the sink causing the network to stop working. Resolving the problem of energy hole is one of the key factors for designing large-scale wireless sensor networks aimed at improving the life span of these systems. Our model in this paper is based on the distribution of working load among the numerous receivers. We have proposed a multiple-sink model for reducing the problem of energy hole via increasing the number of nodes in the vicinity of the sink. This will result in distribution of working load among larger number of nodes in energy consumption bottlenecks of the network. The model consists of different levels of sink intensity, i.e. the number of sinks is determined based on the network's largeness. Finally, we will investigate the proposed model using a numerical analysis.
\end{abstract}

Index Terms-Wireless sensor networks, energy hole problem, multiple-sinks, work load, critical zone.

\section{INTRODUCTION}

A sensor network is composed of a large number of sensor nodes that are densely deployed either inside the phenomenon or very close to it [1]. Sensor nodes are responsible for processing their surroundings and sending the collected data to a specific node called "sink". They are in fact Micro-Electro-Mechanical Systems (MEMS) having limited energy supply (less than $0.5 \mathrm{Ah}$ and $1.2 \mathrm{~V}$ ); these energy consumers are not even rechargeable in most of the wireless sensor network applications due to the nature of their deployment environment [1]. In multi-hop wireless sensor networks (WSNs), each sensor node has the duty to process its own surrounding environment as well as transmitting the packets received from other nodes; therefore, if some part of the sensor nodes is destroyed due to energy depletion it might be disconnected from other nodes causing the network to stop working. Consequently, energy maintenance and management plays a significant role in consistency of these networks.

Energy storage in wireless sensor systems is mainly investigated from 3 aspects: first, using labor-saving hardware pieces particularly in transmitter-receiver block [2], [3], [4]; second, benefiting from energy-effective algorithms

Manuscript received June 21, 2012; revised August 6, 2012.

The authors are with the Department of Technical and Engineering, Islamic Azad University, Tehran North branch, Tehran, Iran (e-mail Mehrdad1986oct@gmail.com). and protocols in software section of the network (e.g. protocol stack) [1], [5], and the last aspect; to take advantage of a balanced energy consumption pattern in all network nodes; otherwise, some parts of the network would run out of energy sooner than others, and if this part is located in crucial zone the network stops working prematurely even if most of the network nodes have high levels of energy [5], [6], [7].

It is not possible to have perfectly balanced energy consumption in WSNs [7]; so, the conducted researches were intended to balance the energy consumption in the nodes to a near-optimal degree [5], [6], [7], [8], [9], [10]. Majority of the researches for decreasing the problem of energy hole are based on the principle of increasing the number of nodes which are directly connected to the sink (e.g. radio radius adjustment approaches [6], strategies of node distribution [7]).

This paper is intended to enlarge the total surface area of the network segment entitled "Critical zone" (more of this in section 3) using several sinks in the network. In this way, we could increase the number of nodes which are responsible for transmitting the working load of the whole network to the sink without adding the computational overhead of nodes [6] or using nodes distribution strategies that are considered as practically difficult tasks [7].

The remainder of this paper is organized as follows: The related work will be briefly reviewed in Section 2 . Section 3 will deal with the problem statement more profoundly. We will present our proposed model in Section 4. The results of our work will be presented in Section 5 via a numerical analysis and finally, we conclude this paper in Section 6.

\section{RELATED WORK}

Authors in [6] investigated enhancement of network's life span based on transmission range adjustment. They modeled the whole network as concentric circles called "Coronas"; the maximal radio radius in each node was divided into " $k$ " levels. Each level equals corona's width; therefore, each node can send forward the data at most up to $\mathrm{k}$ coronas. All nodes in the same corona have the same transmission range and could be differed from transmission range of other coronas. They concluded that the radio radius of each corona shall be chosen optimally in order to resolve the problem of energy hole and to enhance the network's life span. Two algorithms, namely "CETT", "DETL" were applied to obtain the optimized radii.

In [7], the researchers took advantage of non-uniform node distribution for improving the condition of energy hole. The nodes are distributed in a way that the ratio of number of nodes in corona $i+1$ to the nodes in corona $i$ is equal to 
$\frac{(2 i-1)}{q(2 i+1)}$.They inferred that nearly-balanced energy

consumption will be possible in a circular wireless sensor networks provided that the number of nodes increases with the geometrical ratio $q$ from outer layers toward inner ones.

Authors used clustering methods with possibility of changing the cluster heads in [8], [9], and [10] for energy consumption distribution among the nodes. In LEACH [8], all nodes are equally chosen as cluster heads and their selection is done disregarding the nodes' residual energy. In BARC [9], a confidence degree is assigned to each node, cluster heads vary according to the residual energies and confidence degrees. Authors clustered the network efficiently in PEACH [10] without use of overhead resulting from the clustering. Information such as packets of Ads, announcements and connection messages are not used in clusters' configuration.

\section{Problem Statement}

In this section, we are going to get more acquainted with details of energy hole problem. Imagine the network model as illustrated in Fig. 1.

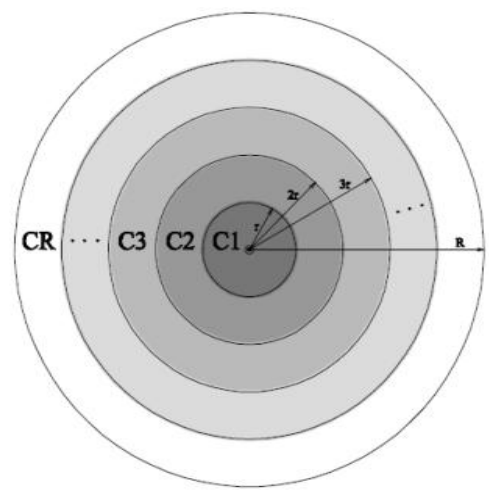

Fig. 1. A circular network model consisting of coronas (Fig. is redrawn from [7]).

\section{UNITS}

The network is divided into concentric circles called "Coronas", in this model, for simplicity, we assume that the radio radius of all nodes equals width of each corona which is equal to 1 unit. The total radio radius of network is designated " $R$ ". Therefore, the network has $R$ coronas notated as $C_{1}, C_{2}, C_{3}, \ldots, C_{R}$ It is assumed that all sensor nodes generate and send $L$ bits of data in each time unit. A large portion of nodes' energies is dissipated in transmitter-receiver block [2], [3], [4]. Thus, we only use consumed energy for sending and receiving data; the energy consumed in other units such as processing, memory, sensing and others are neglected here. It is stated that a node consumes some energy as much as $e_{1}$ for sending 1 bit of data, and consumes as much as $e_{2}$ energy for receiving the same volume of data. $N_{i}$ represents the number of $C_{i}$ nodes, and signifies the consumed energy in time unit for $C_{i}$.

The assumption states that routing causes the data in $C_{i}$ nodes to go forward to $C_{i}-1$ nodes in each hop (in other words, receiving data by the sink is possible via minimal number of hops).

Now, we evaluate the energy consumption in each corona: the outermost corona only transmits its own generated data; thus, the respective energy consumption in time unit is calculated via (1):

$$
E_{R}=N_{R} L e_{1}
$$

All the nodes existing in coronas 1 to $R-1$ send both their own generated packets as well as those received from higher coronas. Accordingly (2), (3) and (4) are respectively used to evaluate the energy consumption for receiving data from higher coronas, energy consumption for sending the received data, and energy consumption for sending the corona's own generated data.

$$
\begin{gathered}
E_{\text {Received }}=L\left(\sum_{k=i+1}^{R} N_{k} e_{2}\right) \\
E_{\text {Transfer }}=L\left(\sum_{k=i+1}^{R} N_{k} e_{1}\right) \\
E_{i}=L N_{i} e_{1}
\end{gathered}
$$

Combination of (1), (2), (3) and 4 will yield (5).

$$
\mathrm{E}_{\mathrm{i}}=\left\{\begin{array}{lc}
N_{\mathrm{R}} \mathrm{Le}_{1}, & \mathrm{i}=\mathrm{R} \\
\mathrm{L}\left[\sum_{\mathrm{k}=\mathrm{i}+1}^{\mathrm{R}} \mathrm{N}_{\mathrm{k}}\left(\mathrm{e}_{1}+\mathrm{e}_{2}\right)+\mathrm{N}_{\mathrm{i}} \mathrm{e}_{1}\right], 1 \leq \mathrm{i} \leq \mathrm{R}-1
\end{array}\right.
$$

According to (5), one can easily Fig. out that the working load imposed by higher coronas results in choking of nodes in the vicinity of sink, specifically the 1 st-Corona which will be designated "critical zone" here forth. In sum, if the nodes of lower coronas are destroyed owing to energy depletion, the links between other nodes of the network are disconnected and as a result, the network stops working. Until now, we have gotten aware of the significance of energy hole problem; the proposed model is investigated in this stage as follows.

\section{OUR PROPOSED MODEL}

The number and the exact position of sink nodes directly affect the life span of WSNs [11]. The number of sinks used in a network is a sort of cost-to-efficiency problem and depends on the network designing criteria. In this regard, our proposed plan is divided into two states, namely: progress in surface and progress in depth. When it is said the network is large; this feature is regarded in two aspects, the geographical region of network is large and there is large number of nodes in it. Combining these states, 3 assumptions can be made regarding the largeness concept:

- Geographical region is large but there is not large number of nodes.

- Geographical region is not large but there is large number of nodes.

- Geographical region is large and there is large number of nodes too. 
Having the above mentioned assumptions, we investigate our recommended solutions:

\section{A. Geographical Region is Large but There is Not Large Number of Nodes}

We consider a base block for our model as indicated in Fig. 2

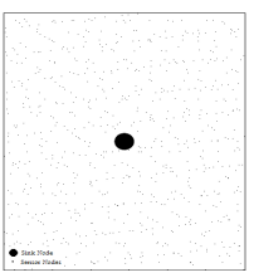

Fig. 2. The base block of our proposed model dark spot is sink node and tiny spots are sensor nodes.

Taking into account the network dimensions, we keep putting together these blocks up to covering the whole network area. This procedure is called "progress in surface". Equation (6) yields us the number of nodes in critical zones of network:

$$
E_{c i}=N \frac{N_{b} \pi r^{2}}{A}
$$

In this equation, $\mathrm{N}$ is total number of sensor nodes, $\mathrm{r}$ is radio radius of sensor nodes, $N_{b}$ represents the number of base blocks and $\mathrm{A}$ is the surface area of whole network.

In large geographical region applications where there is not large number of nodes, another form of energy dissipation occurs in addition to energy hole problem. Due to scattered nodes of the networks, there is a large distance to be traveled by data packets to reach the sink, and consequently, energy loss increases considering the exponential dependence of energy consumption to distance [2], [6]. The number of nodes in critical zones increases by putting together the initial blocks (progress in surface). Thus, the working load is distributed among them; also, the average covered distance from source to destination is reduced via increase in the number of sink nodes.

\section{B. Geographical Region is Not Large but There is Large Number of Nodes Some Common Mistakes}

Our recommended strategy is an aggressive one in this case. In other words, our reaction to large number of nodes is increasing the density of sinks. This time, we try to advance more in depth instead of putting together the initial blocks. This action is designated "progress in depth". We use a grading system to show the level of progress in depth marking them with $D_{1}$ to $D_{n}$ notations. Blocks of $D_{1}$ to $D_{3}$ depths are shown in Fig. 3. (a), Fig. 3. (b) and Fig. 3. (c) respectively.
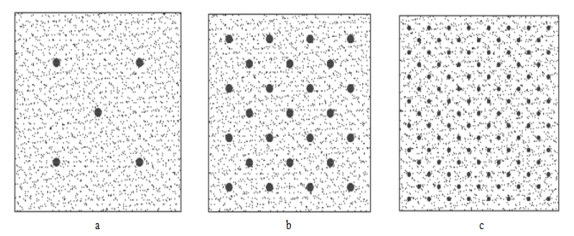

Fig. 3. (a) The base block of $1^{\text {st }}$-depth $(D 1)$, (b) $2^{\text {nd }}$-depth (D2) and (c) $3^{\text {rd }}$-depth $(D 3)$, dark spots are sink nodes. Number of sinks increase in upper levels of progress in depth.
The number of nodes in critical zones of depth " $i$ " can be calculated through (7):

$$
2^{(2 i+1)}-2^{(i+1)}+1
$$

where $N_{S_{i}}$ is the number of sink nodes in depth $i$ in the above equation and is equal to:

\section{Geographical Region is Large and There is Large Number of Nodes Too}

When both geographical region and number of nodes are large, we take benefit of a combination of two former states, i.e. we progress both in depth and surface. The respective network dimensions are covered by putting together some blocks having depths " $i$ ". In this case, the number of sensor nodes in critical zone is obtained via (8):

$$
E_{c_{1}}=N \frac{N_{b} N_{S_{i}} \pi r^{2}}{A}
$$

\section{NUMERICAL RESUlTS AND DISCUSSION}

In this section, we schematically investigate the number of nodes located in critical zone under different conditions of physical dimensions and total number of sensor nodes. Initially, the impact of progress in surface on the number of nodes situated in critical zone will be analyzed and the same effect will be subsequently discussed for progress in depth.

Axes $x$ and $y$ in Fig. 4(a) represent the physical dimensions of network and the number of nodes in critical zones respectively ( $N_{s}$ is the number of sink nodes). As observed in the diagram, whenever physical dimensions of network are enlarged, the network structure having larger number of blocks locates more nodes in the critical zones. For example, for network dimensions of 12000 square meters $d\left(m^{2}\right)$, the number of nodes located in critical zones equals 26 when constructing the network with two adjacent blocks, while this number is nearly 130 in the case of constructing with 10 blocks.

In the diagram of Fig. 4(b), the variations of the number of nodes in critical zone are plotted as we progress in depth.

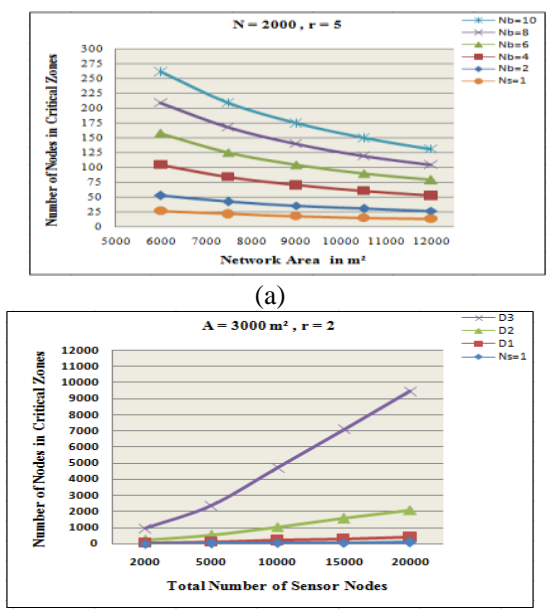

(b)

Fig. 4. (a) Number of nodes in critical zone under large geographical region and invariant total number of nodes, (b) Number of nodes in critical zone under large number of nodes and invariant geographical size of network. 
As indicated in the Fig., when the number of nodes and consequently the working load of network increase, the number of sensor nodes in critical zones also goes up considerably.

The authors in [7] reached to the conclusion that the energy consumption among all network nodes will be nearly balanced via increasing the number of the nodes in the coronas near the sink, and as a result, the life span of wireless sensor networks lengthens. Generally, in most of the conducted researches, it was somehow attempted to increase the number of nodes which were in direct connection with the sink in order to resolve the problem of energy hole. Despite attaining desirable results, some of them included computational overload in sensor nodes (e.g. radio radius adjustment approaches [6]) and other ones were prone to various restrictions (e.g. strategies of node distribution [7]). As an instance, it is observed in node distribution pattern in model [7] that there is a great distance between the nodes of first corona and those of the last one resulting from the increase in the number of network nodes, which makes it very difficult to find an application in real world under such circumstances.

\section{CONCLUSIONS}

In this paper, we proposed a multiple-sink model for reducing the problem of energy hole via increasing the number of sensor nodes around the sink. Our objective was to distribute the generated data packets by nodes to several receivers so as to divide the working load of nodes which are located near the sink onto larger number of sensor nodes. The parts of network which were directly connected to the sink were called "critical zones". The numerical analysis showed how this model leads to increase in the number of sensor nodes in critical zones.

\section{REFERENCES}

[1] I. F. Akyildiz, W. Su, Y. Sankarasubramaniam, and E. Cayirci, "A survey on sensor networks," IEEE Communications Magazine, August 2002, vol. 40, no. 8, pp. 102-114.

[2] A. Depedri, A. Zanella, and R. Verdone, "An Energy efficient protocol for wireless sensor networks," Autonomous Intelligent Networks and Systems (AINS 2003), Menlo Park, CA, Jun-Jul 2003, pp. 1-6.

[3] D. C. Daly and A. P. Chandrakasan, "An energy-efficient OOK transceiver for wireless sensor networks," IEEE Journal of Solid-State Circuits, May 2007, vol. 42, no. 5, pp. 1003-1011.

[4] J. Deng, Y. S. Han, P. Chen, and P. K. Varshney, "Optimal transmission range for wireless ad hoc networks based on energy efficiency," IEEE Transactions on Communications, Sep 2007, vol. 55, no. 9 , pp. $1772-1782$.

[5] J. Li and P. Mohapatra, "Analytical modeling and mitigation techniques for the energy hole problem in sensor networks," Pervasive and Mobile Computing, Jun 2007, vol. 3, no. 3, pp. 233-254.

[6] C. Song, M. Liu, J. Cao, Y. Zheng, H. Gong, and G. Chen, "Maximizing network lifetime based on transmission range adjustment in wireless sensor networks," Computer Communications, July 2009, vol. 32, no. 11, pp. 1316-1325.

[7] X. u, G. Chen, and S. K. Das, "On the energy hole problem of nonuniform node distribution in wireless sensor networks," Mobile Adhoc and Sensor Systems (MASS), Oct. 2006, pp. 180-187.

[8] W. Heinzelman, A. Chandrakashan, and H. Balakrishnan, "An application-specific protocol architecture for wireless microsensor networks," IEEE Transactions on Wireless Communications, Oct. 2002, vol. 1, no. 4, pp. 660-670.

[9] M. K. Watfa, O. Mirza, and J. Kawtharani, "BARC: A battery aware reliable clustering algorithm for sensor networks," Journal of Network and Computer Applications, Nov. 2009, vol. 32, no. 6, pp. 1183-1193.

[10] S. Yi, J. Heo, Y. Cho, and J. Hong, "PEACH: Power-efficient and adaptive clustering hierarchy protocol for wireless sensor networks", Computer Communications, Oct. 2007, vol. 30, no. 14-15, pp. 2842-2852.

[11] E. I. Oyman and C. Ersoy, "Multiple sink network design problem in large scale wireless sensor networks," Communications, June 2004, vol. 6, pp. 3663-3667

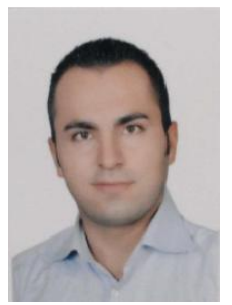

Mehrdad Ahadi received his B.Sc. in computer engineering from Islamic Azad University, Qazvin, in 2008, the M.Sc. in computer engineering from IAU Tehran North Branch, Tehran, in 2011. His research interests are in the areas of distributed systems, ad hoc and wireless sensor networks and evolutionary computing. 